\title{
Measurement of Forces at the Tip of a Cochlear Implant during Insertion
}

\author{
Scott A. Wade, James B. Fallon, Andrew K. Wise, Robert K. Shepherd, Natalie L. James \& Paul R. Stoddart
}

\begin{abstract}
An optical fiber based sensor has been developed to measure the forces at the tip of an electrode array during insertion into the cochlea. The sensor, utilizing optical fiber Bragg grating technology, was incorporated into a custom designed Pt-banded electrode array for guinea pigs. In vivo experiments were undertaken in which forces at the tip of the array were measured in real time during the insertion. Data was obtained for maximum insertion forces of up to $254 \mathrm{mN}$. Histology was performed on the excised cochleae with the sensors fixed in position to evaluate the level of insertion trauma. The insertion experiments demonstrated a clear correlation between applied force and collateral tissue damage.
\end{abstract}

\section{Introduction}

$\mathrm{T}$ HE general aim during the insertion of cochlear implant electrode arrays is to insert the array to an appropriate depth in the cochlea with the electrodes positioned as close as possible to the site of the neural cells in the modiolus [1]. The insertion is performed manually by a surgeon, who stops either at a depth as specified by the manufacturer's insertion procedure or when excessive resistance to the electrode array is encountered. Mechanical trauma to the soft tissues of the nearby spiral ligament and basilar membrane during insertion may cause loss of any residual hearing (see Fig. 1 for more information on cochlear anatomy). This has important implications for speech perception in these patients, as a combination of electrical stimulation and acoustic hearing has been shown to provide better speech perception, particularly in the presence of background noise [2,3]. Moreover, damage to the osseous spiral lamina, the thin sheath of bone separating the spiral ganglion neurons (SGNs) from the scala tympani, will produce a severe loss of SGNs localized to the site of trauma. Finally, electrode insertion trauma will also result in an inflammatory response within the cochlea [4-6] that could result in the loss of SGNs [7] and adversely affect current flow in the implanted cochlea.

Dislocation of the electrode array from the scala tympani into the scala vestibuli may be a further consequence of tearing the basilar membrane, which supports the organ of Corti [8]. This leads to inefficient stimulation of the auditory neurons and has been shown to reduce the patient's ability to process speech after implantation. It may also restrict the use of more advanced speech coding strategies and the potential for the patient to benefit from medical developments or improved surgical procedures that become available in future. In addition, there is some evidence to suggest that the electrode array tends to be only partially inserted when the surgeon perceives resistance during insertion. Incomplete insertion is associated with reduced clinical performance [9]. These negative indications are by no means inevitable. Animal studies have shown that the delicate hair cells close to the electrode can survive the implantation, provided that electrode insertion trauma and severe inflammation is avoided [10].

A variety of approaches have been considered in order to address the issue of trauma during cochlear implant insertion [11]. Studies have shown that tearing of delicate cochlear tissues can occur when the tip of the electrode array has been inserted 7-11 mm into the cochlea $[12,13]$ and again at about $14 \mathrm{~mm}$ onwards [14]. Researchers have attempted to study the mechanical behavior of the electrode by means of a 3-D finite element analysis of the cochlea $[15,16]$. For straight electrode array designs, these studies confirm that the resistance to insertion starts to increase from about $5 \mathrm{~mm}$ and peaks after approximately $8-10 \mathrm{~mm}$, which corresponds to the point where the electrode tip encounters the back wall of the cochlea [16]. A numerical simulation based on a more realistic 3D model of the scala tympani suggests that the damage at $14 \mathrm{~mm}$ may be associated with a characteristic change in inclination of the scala tympani spiral at approximately $10 \mathrm{~mm}$ [15]. Rotational tomography has also been used to visualize the position of the array after surgery [8]. These results have helped to identify improved surgical techniques and have allowed different electrode array designs that minimize the trauma during insertion [17]. An interesting alternative design involves the use of steerable electrode arrays that can actively bend at the tip via an embedded strand [18].

Another initiative involves the development of tools to assist the surgeon in accurately positioning the electrode array at the "round window" entrance to the cochlea for final insertion. This requires high resolution 3D imaging of the skull and the use of a robotic tool to precisely measure the shape of the ear [19]. An optical imaging probe may be used to map the fine structure of the inner ear. It is expected that this combination of technologies will allow a computer-controlled instrument to drill the insertion channel through the petrosal bone and the middle ear to the precise target location at the round window on the lower cochlear coil [20]. The risk of damage to intact sensory cells may, for example, be reduced if the electrode array can be positioned to a high level of initial accuracy. Although these kinds of visualization and guidance tools are likely to assist in the initial part of the procedure, real-time feedback remains critical for controlling the dynamic behavior of the array during insertion into the cochlea and thereby reducing the risk of trauma in individual situations. 
Recent work on automated insertion has seen the integration of force sensing in the robotic insertion tool [21-23].

Another area being investigated to avoid trauma has been in the development of sensors for use in cochlear implants. Most of the efforts to date have been directed towards monitoring the depth of implantation or the position of the implant within the cochlea. Perhaps the most ambitious approach has used polycrystalline diamond as a piezoresistive position sensor on a thin film electrode array with high lateral stiffness [24]. Polycrystalline-silicon was used as the piezoresistive strain gauge material in subsequent work [25, 26]. Eight segmented piezoresistive strain gauges were used to measure the local deflection of the array, allowing overall array shape to be determined to better than $50 \mu \mathrm{m}$. A piezoresistive tip sensor was used to detect wall contact, but the relationship between tip sensor voltage and force has not been quantified in this or any subsequent work.

The features of position detection and wall contact sensing were expected to improve the depth of insertion and reduce insertion damage. Shape control could potentially be achieved by means of an articulated insertion tool, an inserted wire stylet or by pressurized fluid actuation [27]. This work could eventually constitute a first step toward providing closed-loop control of array insertion [28].

Optical fiber sensors have been developed to measure a wide range of sensing parameters. Small size, light weight and immunity to electromagnetic interference are but a few of the advantages that they have over corresponding technologies [29]. Many of these attributes also make optical fiber sensors an attractive option for use in cochlear implants. For example, $\mathrm{Li}$ and Yao developed an optical fiber bending sensor, which used bend-induced losses of reflected light to infer the shape of the electrode array [30]. They concluded that this sensor provided a promising technique for in situ monitoring of the shape and position of the array during the implantation procedure. However, the fiber response is ambiguous under inhomogeneous bending conditions, in the sense that a long bend of large radius may cause the same loss as a short bend of small radius.

This paper describes the development of an optical fiber force sensor that is integrated into the electrode array of a cochlear implant. The sensor, based on optical fiber Bragg grating (FBG) strain sensors, was used to measure and display in real time, the forces exerted by the tip of an electrode array on the tissues of guinea pig cochleae during insertion. Apart from providing information about tissue damage thresholds during insertion, the sensor should help to establish whether force feedback could be used by a surgeon to minimize mechanical trauma in real time during surgery.

\section{Materials and Method}

\section{A. Optical Fiber Force Sensor}

The force sensor described in this work used an optical fiber Bragg grating. These in-fiber devices have been used extensively in telecommunications and sensing applications [31]. They consist of a periodic modulation of the refractive index in the core of the optical fiber. When the wavelength of light propagating in the fiber is phase matched with the periodicity of the modulation, a narrow band of light will be reflected. Therefore any external parameter, such as strain, temperature or pressure, which changes the effective period of the refractive index modulation, will cause a shift in the reflected wavelength. Consequently sensors based on FBGs have been applied in a range of applications, such as fiber optic catheters for the diagnosis of gastrointestinal motility disorders [32].

The FBGs used in this work were written in the $1550 \mathrm{~nm}$ wavelength region in $\mathrm{H}_{2}$ pre-sensitized, standard telecommunications fiber $(9 / 125 \mu \mathrm{m}$ core-cladding diameter). A scanning phase mask technique was used with $244 \mathrm{~nm}$ radiation from a frequency-doubled argon-ion laser. Typical FBG parameters were $2 \mathrm{~mm}$ length, $10 \mathrm{~dB}$ reflectance and full width half maximum bandwidth of $0.5 \mathrm{~nm}$.

The choice of parameters for the Bragg grating used in the sensor was guided by experience obtained during proof of concept tests and modeling. The aim was to develop a system that was able to measure over several hundred $\mathrm{mN}$ of force, with a relatively small sensor located at the fiber tip. Bragg gratings with a length of $2 \mathrm{~mm}$ proved to be a good compromise between sensing bandwidth (as determined by the full width half maximum of the FBG reflectance), sensor length and grating reflectivity.

A wide variety of methods exist to monitor the shift in wavelength of a FBG due to an external perturbation. In this work we used a relatively low cost matched FBG interrogation scheme that was designed and built by the authors. In this technique the light signal reflected from the sensor FBG is passed through a second "filter" FBG which has a center wavelength slightly offset to the sensor grating. The amount of light transmitted by the filter FBG depends on the wavelength of the sensor FBG and hence the force exerted on the probe. Temperature control of the filter FBG allowed optimization of the center wavelength offset between the sensor and filter Bragg gratings during a test. This transmission mode interrogation system used a second "reference" signal to compensate for any background intensity fluctuations. The measurement range and accuracy of the FBG monitoring system depends somewhat on the characteristics of the FBGs used [33]. For the purposes of this work the system was designed to provide a force measurement range on the order of several hundred milliNewtons $(\mathrm{mN})$ with sub-mN accuracy. While not tested, the maximum measurement frequency of the unit was only limited by the analog-to-digital hardware used (USB Instruments, DS1M12, $1 \mathrm{MS} / \mathrm{s}$ native sampling rate). Further details of the interrogation scheme can be found in reference [33]. In addition to standard sensor output recording a software program was used to display real-time sensor measurements. 


\section{B. Sensor probe design}

The FBGs used in this work were written in the center of a length of optical fiber using a standard phase mask writing technique. Gratings were characterized by means of a JDS Uniphase SWS15100 swept wavelength system (3 pm resolution). The sensor probes were then fabricated by cleaving the fiber close $(\sim 1 \mathrm{~mm})$ to one end of the FBG. Measurements of the FBG reflection spectra were performed after the cleaving step to confirm that the procedure had not affected the key sensor characteristics.

For the animal experiments the force sensor optical fibers were incorporated into a custom designed guinea pig platinumbanded electrode array [34, 35]. This array is similar in design principle to the Cochlear Ltd. banded electrode arrays. The array consisted of three platinum ring electrodes $(0.2 \mathrm{~mm}$ wide; $0.4 \mathrm{~mm}$ diameter; $0.4 \mathrm{~mm}$ inter-electrode spacing) mounted in Silastic (MED1-4213, NuSil), with each electrode welded to a parylene insulated platinum/iridium wire $(25 \mu \mathrm{m}$ diameter). A fourth 'dummy' platinum ring was located $\sim 4.5 \mathrm{~mm}$ from the tip of the array and was used as a guide to insertion depth. Prior to embedding the wired platinum rings in Silastic, the force sensor fiber containing the FBG was threaded through the platinum rings so that the tip of the optical fiber was $\sim 0.2 \mathrm{~mm}$ past the outer edge of the platinum ring closest to the tip of the array. The injection moulding process used to fabricate the array also helped to ensure that the optical fiber was located close to the center of the cylindrical array. This configuration has similarities to the arrangement reported in [36]. Fig. 2 shows a schematic of the electrode array containing the force sensor.

\section{Insertion Experiments}

Six young healthy adult pigmented guinea pigs of either sex (300-600 g, 4-9 months old) were used in total for the experiments reported. All procedures were approved by the Animal Research Ethics Committee of the Royal Victorian Eye and Ear Hospital in accordance with the National Institute of Health (NIH) Guidelines for the Care and Use of Laboratory Animals and conformed to the Code of Practice of the National Health and Medical Research Council of Australia.

All experiments were performed on anaesthetized animals (ketamine, $60 \mathrm{mg} / \mathrm{kg}$ and xylazine, $4 \mathrm{mg} / \mathrm{kg}$; intramuscular [i.m.]). Anaesthesia was maintained with xylazine $(1 \mathrm{mg} / \mathrm{kg})$ in ketamine (10 mg/kg; i.m.) every $40-50 \mathrm{~min}$ or as required as determined by a pedal withdrawal reflex. The external meatus and tympanic membrane of each animal was examined otoscopically and determined to be normal. Body temperature was maintained at $37{ }^{\circ} \mathrm{C}$ with a homeothermically regulated heat pad. Lignocaine was injected subcutaneously around the ear canals and in any skin that was to be incised. The head was immobilized in a custom head holder. Using standard surgical techniques, the round window of the left and right cochlea were exposed via a post-auricular approach, followed by a cochleostomy (diameter $\sim 1 \mathrm{~mm}$ ) to allow access to the scala tympani (where the array was inserted) [37].

The custom electrode arrays containing the optical fiber force sensors were glued to a quartz glass micropipette that was mounted on a 2662 Micropositioner (Kopf, USA), see Fig. 2. Prior to the insertion of the individual electrode arrays containing force sensors, several temperature calibrations were performed, with the array sensor located in the middle ear cavity and located within the cochlea (both with and without perilymph). This calibration was carried out by scanning the temperature of the filter FBG which allowed temperature corrections to be made and the sensitivity of the sensor to be determined.

Prior to force measurements the position of the electrode array was set by placing the tip of the array at the cochleostomy, with the visual contact of the tip with perilymph used to indicate this location. This position at the opening of the cochlea was set as the zero point on the micropositioner and used as a reference distance for all subsequent insertion depth measurements. The force sensor array was withdrawn a set distance of $2 \mathrm{~mm}$ from the zero point ready for insertion. Fig. 3 illustrates the direction of the insertion of the electrode array into the cochlea and the location of the FBG force sensor at the tip of the array.

The recording electronics of the force sensor were started, with a sampling rate of up to $25 \mathrm{~Hz}$, and allowed to record the baseline signal level for a period of up to $30 \mathrm{~s}$. The electrode array was then advanced into the cochlea at a controlled rate of $100 \mu \mathrm{m} / \mathrm{s}$. This insertion speed was chosen to allow a reasonable level of control of the insertion depth, which was stopped manually. The insertion trajectory was chosen so that the array impacted the lateral wall of the scala tympani in the upper basal turn. In order to measure forces during insertion, some of the experiments were designed to ensure that some level of trauma occurred; hence the insertion was often deeper than would normally occur.

The output of the force sensor and video footage of the insertion were simultaneously recorded to allow for offline reconstruction of the insertion experiment. The depth at which insertion was stopped was determined by a number of parameters which were specific to the requirements of three separate sets of experiments. The first experiment was designed to examine the difference between insertions performed to a depth of either $1.5 \mathrm{~mm}$ or $5 \mathrm{~mm}$ past the cochleostomy opening. These depths were chosen to represent cases where minimal trauma and some level of damage occurred during the insertion. In the second experiment all insertions were stopped after a $3.5 \mathrm{~mm}$ insertion past the cochleostomy opening. This depth was found to produce damage to cochlear structures in the first experiment. The insertions were stopped manually by an operator using a digital display for feedback, which provided an accuracy of $\pm 0.1 \mathrm{~mm}$. The third and final experiment was similar to the first and aimed to investigate the difference between a traumatic insertion and an insertion where minimal trauma would occur. In this case however the insertions were controlled using a maximum force, as measured in real-time by the sensor, to determine when the insertion was to be stopped. Values of atraumatic and traumatic force levels were estimated 
using the data from the previous sets of insertion experiments to be $75 \mathrm{mN}$ and $225 \mathrm{mN}$, respectively (see Discussion). In the third experiment, the two guinea pigs were tested with a traumatic insertion in one cochlea and an atraumatic insertion in the other. In each of the three experiments, insertions were performed on both cochleae of two individual guinea pigs. This gave four data points per experiment.

At the end of each insertion the electrode array was secured in position within the cochlea by fixing it in two locations. The guinea pig was then given an overdose of Lethabarb (150 $\mathrm{mg} / \mathrm{kg}$; intraperitoneal) and transcardially perfused with warm $0.9 \% \mathrm{NaCl}$ followed by $4{ }^{\circ} \mathrm{C} 10 \%$ neutral buffered formalin $(\mathrm{NBF})$ in $0.1 \mathrm{M}$ phosphate buffer saline solution $(\mathrm{pH}=7.35)$. The stapes was displaced from the left and right oval windows, the bone at each apex was opened, and the left and right round windows were punctured to facilitate the diffusion of fluids through the cochleae. The cochleae were kept in $10 \%$ NBF for post-fixation overnight at $4{ }^{\circ} \mathrm{C}$, after which histological processing and examination took place. Specimens were dehydrated in ethanol using serial concentrations progressing from 70 to $100 \%$ and immersed in degassed epoxy resin for acrylic fixation. Vacuum was applied to ensure that the epoxy mixture fully infiltrated the cochlea. After embedding the specimens were X-rayed to assess the correct plane for sectioning. Excess portions of the temporal bone within the epoxy blocks were trimmed down to the cochlea. These specimens were re-embedded with the orientation of the blocks aligned such that the central axis of the cochlear was parallel to the plane of sectioning, perpendicular to the electrode array [38].

The cochlear specimens were serially sectioned using a grinding technique, with a section thickness that was adapted for specific experiments; in experiment \#1 sections were $200 \mu \mathrm{m}-300 \mu \mathrm{m}$; in experiment \# 2 sections were $300 \mu \mathrm{m}$ and in experiment \#3 sections were $200 \mu \mathrm{m}$. Each section was polished prior to staining with toluidine blue and examination under light microscopy with photography.

The level of trauma observed in histological specimens as a result of individual insertion tests was graded using blind techniques by applying a modified version of the scale reported by Eshraghi et al. [39]:

- 0 - no observable trauma,

- 1 - elevation of the basilar membrane,

- 2 - rupture of basilar membrane or tear of the spiral ligament, and,

- 3 - fracture of the osseous spiral lamina or modiolus.

See Fig. 1 for information on the relevant anatomy of the cochlea. The main differences between this scale and that of Eshraghi et al. are that tearing of the spiral ligament has been added to category 2, category 3 has been removed as we saw no electrodes in the scala vestibuli and the severest trauma category has been simplified. Unless otherwise specified a trauma grading level was categorized if present in at least two consecutive sections.

\section{Results}

\section{A. Force sensor calibrations}

In order to test the performance of the sensor unit, and before any use in animal experiments, force and temperature calibrations were performed in the laboratory. Axial force calibrations were performed by pressing the tip of the array containing the force sensor at $90^{\circ}$ onto a high accuracy mass balance for a fixed period, recording the sensor output and converting the indicated mass to an equivalent force. The force level was then increased and another set of readings were taken. This process was repeated to cover the range of forces expected in the testing, typically up to a few hundred $\mathrm{mN}$. These calibrations were repeated a number of times on each sensor. The temperature of both the sensor and filter Bragg gratings were recorded during calibrations to allow correction for any thermal drift.

In addition to force calibrations, the effect of temperature on the sensor output was measured for individual sensor probes. This information was required for compensation of the sensor measurements for the shift from room temperature to body temperature during implantation. Compensation was obtained by controlling the temperature of the filter FBG with a Peltier device, as the sensor measurement is only affected by the relative difference in temperature between the filter and sensor FBGs. In a manner similar to the force calibrations, data was recorded at a series of discrete temperature levels.

The sensor interrogation system measures the output of two photodiodes, the ratio of which is used to calibrate the Bragg grating sensor. Examples of force and temperature calibrations are shown in Fig. 4, where the sensor data was averaged over $10 \mathrm{~s}$ during multiple cycles of increasing levels of static force or temperature. The average RMS error for the force calibration data shown was $0.39 \mathrm{mN}$, calculated using the error in the ratio measurements which was then converted to an equivalent force.

\section{B. Cochlear insertion experiment \#1}

Examples of the raw forces measured during traumatic and atraumatic insertions are shown in Fig. 5. As discussed in Section IIC, the insertion used in this work differed somewhat from that used in standard insertions. Therefore the insertion distance and the data in the following force figures should not be compared with those obtained during standard electrode array insertions. The small decrease in the forces at the start of insertion shown in Fig. 5 are believed to be due to a slight temperature increase as the force sensing electrode array is inserted deeper into the guinea pig cochlea and reflects the difference between peripheral and core temperatures in the guinea pig. This drift was also observed in the remaining insertion tests, with a maximum value of approximately -10 $\mathrm{mN}$. This drift was small relative to the magnitude of forces measured and therefore was deemed acceptable for this application.

The aim of this experiment was to stop the traumatic insertions at a depth of $+5 \mathrm{~mm}$, however in two cases the insertions were stopped before this depth due to significant bending of the sensor probe being observed, in both cases the 
insertion resulted in trauma to the cochlea. The maximum forces measured during the four sensor array insertions are given in Table I. As expected no obvious force was measured at the electrode array sensor tip during the atraumatic insertions (1-a and 1-c).

Histological micrographs showing sections of the force sensors fixed in their final insertion position are shown in Fig. 6, and illustrate both a traumatic insertion (Fig. 6(a)) and an atraumatic insertion (Fig. 6(b)). Using the set of histological images for each insertion, trauma grades were determined, the results of which are given in Table I.

\section{Cochlear insertion experiment \#2}

The forces measured during the four insertion tests of the second experiment are shown in Fig. 7, as a function of insertion distance. For each of these tests the insertions went slightly past the intended maximum insertion depth of +3.5 $\mathrm{mm}$. A clear difference is seen in the maximum forces measured for the two animals, with higher forces being measured in the tests on the first guinea pig in this experiment (i.e. insertions 2-a and 2-b). The maximum forces for each of the four insertions are provided in Table I. Fig. 7 also shows that the insertion depth at which the contact force starts to increase differs slightly among the individual insertion tests. This may be due to several variables including slight errors in determining the zero point for insertion, variability in the position and size of the cochleostomy, angle of insertion, variability in positioning the array at the opening of cochleostomy or differences in sizes of the cochlea. This does however illustrate the potential advantages of real time force feedback in minimizing electrode array insertion trauma.

Examples of histological specimens taken with force sensors arrays fixed in the final insertion positions are given in Fig. 8. The images provide an example from an insertion test performed in each of the two guinea pigs tested in this experiment. The maximum force measured in the insertion shown in Fig. 8 (a) was 2.5 times greater than that recorded during the experiment shown in Fig. 8 (b). Gradings of the estimated trauma damage observed in the histological images of the four insertion tests are given in Table I. The insertion from 2-b was given a grade 2-3 (one image with clear evidence of trauma at a grade 3 level).

\section{Cochlear insertion experiment \#3}

In the third insertion experiment the aim was to stop the insertion at a force of either $75 \mathrm{mN}$ or $225 \mathrm{mN}$, forces that were determined in the previous experiments to be atraumatic and traumatic respectively. The actual maximum forces for each insertion, as listed in the lower section of Table I, varied slightly from the target forces due to the response time of the operator, but were deemed to be acceptable for the purposes of the experiment. The changes in force measured at the sensor array tip as a function of insertion distance are shown in Fig. 9. Again the depth at which the contact forces begin to rise varies across the different insertions performed.

In Fig. 10 examples of histological specimens from two insertions are illustrated. The top image is for one of the higher force insertions $(225 \mathrm{mN})$, while the bottom image is from one of the lower force insertions $(75 \mathrm{mN})$. The trauma grades for each of the insertion tests in this experiment are listed in Table I. The two insertions with lower maximum force gave trauma grades of 0 and 2, while the insertion with maximum forces of $\sim 225 \mathrm{mN}$ both produced trauma grades of 3 .

A graphical representation of the maximum insertion forces and corresponding trauma grades is provided in Fig. 11. The relatively small number of insertions does not provide conclusive information on the maximum forces that can be tolerated in order to avoid specific types of trauma. The data however does show a definite trend of increased levels of trauma as the maximum insertion force increased.

\section{Discussion of Results}

In general there appears to be a lack of data examining the mechanical properties of bony structures and soft tissues in the cochlea in relation to cochlear implantation. The forces against the outer wall of the cochlea have been measured with an Instron load cell during the insertion of an electrode array in cochlear models and in formalin-fixed temporal bones [17]. Data from a Teflon cochlear model showed that the "advance off stylet" technique with the Advance electrode array generated peak forces of only $10 \mathrm{mN}$, whereas the Contour electrode array developed forces of 70 to $110 \mathrm{mN}$. Higher loads of up to $240 \mathrm{mN}$ were measured in the temporal bones under the same conditions for the standard insertion technique, although the general form of the force versus array displacement was the same in both cases.

A key study by Ishii et al. [40] used a needle connected to semiconductor force sensors to measure the mechanical properties of cochlear tissues taken from autopsied cases. The rupture force was $564 \mathrm{mN}$ for the round window membrane, 26-35 $\mathrm{mN}$ for the basilar membrane and only $4.2 \mathrm{mN}$ for Reissner's membrane. The corresponding elastic (Young's) moduli were 9.8 $\mathrm{MPa}$ for the round window membrane, 6.09.7 $\mathrm{MPa}$ for the basilar membrane and $34.2 \mathrm{MPa}$ for Reissner's membrane. The authors note that there is no absolute value for the mechanical properties of biological materials, with parameters such as the tested size of the specimen, the sensor needle geometry and the displacement speed influencing the results [40].

While it is difficult to create reproducible experimental conditions in these ex vivo studies, the force measurements are in reasonable agreement with the present results obtained in vivo at the tip of an array during the insertion of an electrode array. The fiber Bragg grating based sensor, together with lowcost, high-sensitivity interrogation unit developed in this work, provides a resolution of approximately $1 \mathrm{mN}$, which is equivalent to $0.08 \mathrm{MPa}$ (for the $125 \mu \mathrm{m}$ diameter optical fibre used). The sensor is thermally stable under operational conditions, showing a maximum drift of $\sim 10 \mathrm{mN}$ as it was inserted deeper into the guinea pig cochlea. As this drift is in the opposite direction to the response under compression, it was deemed acceptable in the current context. If required a number of methods to correct for the temperature crosssensitivity can be used (e.g. [41, 42]). The system provides a 
real-time display of sensor output (force) with a sampling rate of up to $100 \mathrm{kHz}$. This can easily be modified as required to provide a more convenient interface or feedback mechanism for the surgeon during implantation.

One of the aims of the current work was to investigate if there was any correlation between the maximum level of contact force that occurs during the insertion of an array and subsequent structural damage. With the first two experiments however the insertion depth was used as a control in the tests and as a result the peak forces were not fully controlled. Future experiments investigating the level of trauma would best be performed using the measured maximum force to determine the final insertion depth, as used in the final experiment. It is important to reiterate that due to the limited number of animals tested in this work, further trials are required before definitive correlations between the level of insertion force and subsequent trauma can be made.

It has recently been shown that for straight electrode arrays the frictional interaction between the electrode array and the wall of the cochlea depends on the insertion speed [43]. Previous studies of the forces applied during electrode array insertion by others have been carried out using a range of insertion rates (e.g. $0.3 \rightarrow 2 \mathrm{~mm} / \mathrm{s}$ ) [44-46], while a recent study evaluating the insertion rate of electrode arrays of a number of surgeons reported a mean insertion rate of $1.6 \mathrm{~mm} / \mathrm{s}$ [47]. Therefore, it may be interesting to investigate how the insertion rate affects the resulting force measured with the electrode array containing the force sensor. Future work may also involve adding periods in which the electrode/sensor is stationary to allow thermal stabilization.

Various studies have used three-point flexural bending and buckling tests to determine stiffness properties of electrode arrays [48, 49]. Lim et al. [16] have reported elastic moduli for compact bone (20 GPa), keratin (3 GPa), basilar membrane fibers $(1.9 \mathrm{GPa})$ and basilar membrane ground substance $(0.2$ $\mathrm{MPa}$ ), but it is not clear what source they use for this data. In comparison, the elastic moduli of common electrode array materials were reported as $8.27 \mathrm{GPa}$ for $\mathrm{Pt} / \mathrm{Ir} / \mathrm{PTFE}$ used for wires and $0.45 \mathrm{MPa}$ for silicone elastomer. In comparison, the modulus for an optical fiber is approximately $72.5 \mathrm{GPa}$ for fused silica [50]. Indeed, deflection force measurements of an electrode array containing an embedded $125 \mu \mathrm{m}$ diameter fiber were $\sim 6$ times higher than those for a standard guinea pig array without an embedded optical fiber. From this it is clear that the $125 \mu \mathrm{m}$ diameter optical fiber dominates the mechanical properties of the array.

The current sensor is primarily designed to measure the axial forces at the tip of a cochlear implant during insertion. Forces applied by the tip of an electrode are considered to be responsible for one of the most common types of insertion injury, namely penetration of the basilar membrane and movement of the electrode into the scala vestibuli $[14,51]$. Hence an understanding of the forces applied at the tip of the electrode implant is critical to developing insertion techniques and implant designs that reduce trauma. It is important to note however that there are a number of forces at play during insertion, including axial and lateral forces at the tip, frictional forces and lateral forces from the proximal section of the array $[46,52]$. In most of the studies of insertion forces reported to date, the experimental configuration measured a combination of these forces [17, 21, 47, 53], which does not provide complete information on all of the individual force components. One of the areas for future study with the proposed sensor could be to obtain detailed information on the individual force components at play during the insertion. There are a number of other potential improvements to the proposed sensor design that could be explored, such as minimizing the sensor FBG length to optimize the spatial selectivity of the region being monitored.

In all of the animal experiments undertaken here, sensors with Bragg gratings written in $125 \mu \mathrm{m}$ fiber were used. This diameter fiber was chosen for use as it is a standard diameter in many telecommunications systems and therefore compatible handling tools and components are relatively cheap and readily available. In order to reduce the array stiffness, it may be possible to use a smaller diameter fiber, such as $80 \mu \mathrm{m}$, for the sensor. This may allow deeper insertion of the sensor into the cochlea. Some other potential advantages of smaller diameter fiber sensors include increased force sensitivity and easier incorporation into the electrode array.

\section{Conclusion}

Insertion experiments performed with the force sensor on anaesthetized guinea pigs have demonstrated a correlation between applied force and tissue damage in the guinea pig cochlea. The results obtained indicate that this sensor could be used to study the mechanical properties of delicate cochlear structures in vivo. This information is potentially useful for mechanical modeling of the cochlear and could be used to direct future improvements in array design [51]. However, there is a limit to the information that can be obtained from a uniaxial sensor in a complex 3D geometry such as the cochlea.

The results of this study also serve to highlight the challenges of manual insertion. After the array made contact with the back wall of the cochlear spiral, a rapid increase in force occurred within about $0.2 \mathrm{~mm}$. Considering typical implant insertion rates and relatively slow human reaction times, it may be difficult for a surgeon to effectively use the sensor feedback to minimize trauma. However, the device could potentially be used for training purposes or form part of an automated insertion tool that offers an increased level of motion control [21].

\section{Acknowledgment}

The authors would like to thank Irfan Durmo (Cochlear Ltd) for assistance with histology, and Dr Sam Irving and Helen Feng (Bionics Institute) for assistance with aspects of the in vivo experiments and electrode array fabrication, respectively. 


\section{References}

R. K. Shepherd, S. Hatsushika, and G. M. Clark, "Electrical stimulation of the auditory nerve: the effect of electrode position on neural excitation," Hear. Res., vol. 66, pp. 108-20, Mar 1993.

[2] B. J. Gantz and C. W. Turner, "Combining acoustic and electrical hearing," Laryngoscope, vol. 113, pp. 1726-1730, Oct 2003.

[3] J. Kiefer, M. Pok, O. Adunka, E. Sturzebecher, W. Baumgartner, M. Schmidt, et al., "Combined electric and acoustic stimulation of the auditory system: results of a clinical study," Audiol. NeuroOtol., vol. 10, pp. 134-144, 2005.

[4] J. Xu, R. K. Shepherd, R. E. Millard, and G. M. Clark, "Chronic electrical stimulation of the auditory nerve at high stimulus rates: A physiological and histopathological study," Hear. Res., vol. 105, pp. 1-29, Mar 1997.

[5] P. Wardrop, D. Whinney, S. J. Rebscher, W. Luxford, and P. A. Leake, "A temporal bone study of insertion trauma and intracochlear position of cochlear implant electrodes. II: Comparison of Spiral Clarion and HiFocus II electrodes," Hear. Res., vol. 203, pp. 68-79, 2005.

[6] R. J. Vivero, D. E. Joseph, S. Angeli, J. He, S. Chen, A. A. Eshraghi, et al., "Dexamethasone base conserves hearing from electrode trauma-induced hearing loss," Laryngoscope, vol. 118, pp. 2028-2035, 2008.

[7] P. A. Leake, G. T. Hradek, and R. L. Snyder, "Chronic electrical stimulation by a cochlear implant promotes survival of spiral ganglion neurons after neonatal deafness," The Journal of Comparative Neurology, vol. 412, pp. 543-562, 1999.

[8] A. Aschendorff, J. Kromeier, T. Klenzner, and R. Laszig, "Quality control after insertion of the nucleus contour and contour advance electrode in adults," Ear Hear., vol. 28, pp. 75S-79S, Apr 2007.

[9] M. Neumann, A. Aschendorff, J. Schipper, R. Laszig, and T. Klenzner, "The influence of insertion depth on the preservation of residual hearing after cochlear implantation," Laryngo-RhinoOtol., vol. 84, pp. 113-116, Feb 2005.

[10] A. Coco, S. B. Epp, J. B. Fallon, J. Xu, R. E. Millard, and R. K. Shepherd, "Does cochlear implantation and electrical stimulation affect residual hair cells and spiral ganglion neurons?," Hear. Res., vol. 225, pp. 60-70, Mar 2007

[11] B. S. Wilson, D. T. Lawson, J. M. Muller, R. S. Tyler, and J. Kiefer, "Cochlear implants: Some likely next steps," Annu. Rev. Biomed. Eng., vol. 5, pp. 207-249, 2003.

[12] B. K.-H. G. Franz and G. M. Clark, "Refined surgical technique for insertion of banded electrode array," Ann. Otol. Rhinol. Laryngol., vol. 96, pp. 15-17, 1987.

[13] R. K. Shepherd, G. M. Clark, B. C. Pyman, and R. L. Webb, "Banded intracochlear electrode array: evaluation of insertion trauma in human temporal bones," Ann. Otol. Rhinol. Laryngol., vol. 94 , pp. 55-59, 1985.

[14] P. Wardrop, D. Whinney, S. J. Rebscher, J. T. Roland, W. Luxford, and P. A. Leake, "A temporal bone study of insertion trauma and intracochlear position of cochlear implant electrodes. I: comparison of Nucleus banded and Nucleus Contour electrodes," Hear. Res., vol. 203, pp. 54-67, 2005.

[15] H. N. Kha, B. K. Chen, and G. M. Clark, "3D finite element analyses of insertion of the Nucleus standard straight and the Contour electrode arrays into the human cochlea," J. Biomech. vol. 40, pp. 2796-2805, 2007.

[16] Y. S. Lim, S. I. Park, Y. H. Kim, S. H. Oh, and S. J. Kim, "Threedimensional analysis of electrode behavior in a human cochlear model," Med. Eng. Phys., vol. 27, pp. 695-703, Oct 2005.

[17] J. T. Roland, "A model for cochlear implant electrode insertion and force evaluation: Results with a new electrode design and insertion technique," Laryngoscope, vol. 115, pp. 1325-1339, Aug 2005

[18] J. A. Zhang, J. T. Roland, S. Manolidis, and N. Simaan, "Optimal path planning for robotic insertion of steerable electrode arrays in cochlear implant surgery," J. Med. Devices, vol. 3, Mar 2009.

[19] J. Schipper, A. Aschendorff, I. Arapakis, T. Klenzner, C. B. Teszler, J. Ridder, et al., "Navigation as a quality management tool in cochlear implant surgery," J. Laryngol. Otol., vol. 118, pp. 764-770, Oct 2004.

[20] R. F. Labadie, O. Majdani, and J. M. Fitzpatrick, "Image-guided technique in neurotology," Otolaryngol. Clin. N. Am., vol. 40, pp. 611-624, Jun 2007.

[21] D. Schurzig, R. F. Labadie, A. Hussong, T. S. Rau, and R. J. Webster, "Design of a tool integrating force sensing with automated insertion in cochlear implantation," IEEE-ASME Trans. Mechatron., vol. 17, pp. 381-389, Apr 2012.

[22] D. Schurzig, R. J. Webster, M. S. Dietrich, and R. F. Labadie, "Force of cochlear implant electrode insertion performed by a robotic insertion tool: comparison of traditional versus advance off-stylet techniques," Otol. Neurotol., vol. 31, pp. 1207-1210, Oct 2010 .

[23] J. Zhang, W. Wei, J. Ding, J. T. Roland, S. Manolidis, and N. Simaan, "Inroads toward robot-assisted cochlear implant surgery using steerable electrode arrays," Otol. Neurotol., vol. 31, pp. 1199-1206, 2010.

[24] Y. X. Tang, D. M. Aslam, J. B. Wang, and K. D. Wise, "Technology and integration of poly-crystalline diamond piezoresistive position sensor for cochlear implant probe," in Transducers '05, Digest of Technical Papers, Vols 1 and 2, 2005, pp. 543-546.

[25] J. Wang, A. Gulari, and K. D. Wise, "An integrated positionsensing system for a MEMS-based cochlear implant," in IEEE International Electron Devices Meeting 2005, Technical Digest, 2005, pp. 129-132.

[26] J. B. Wang, M. N. Gulari, and K. D. Wise, "A parylene-silicon cochlear electrode array with integrated position sensors," presented at the 28th Annual International Conference of the IEEE Engineering in Medicine and Biology Society, Vols 1-15, 2006.

[27] K. D. Wise, P. T. Bhatti, J. B. Wang, and C. R. Friedrich, "Highdensity cochlear implants with position sensing and control," Hear. Res., vol. 242, pp. 22-30, Aug 2008.

[28] J. B. Wang and K. D. Wise, "A thin-film cochlear electrode array with integrated position sensing," J. Microelectromech. Syst., vol. 18, pp. 385-395, Apr 2009.

[29] J. M. López-Higuera, Handbook of Optical Fiber Sensing Technology: Wiley, 2002.

[30] E. B. Li and J. Q. Yao, "Fiber-optic bending sensor for cochlear implantation - art. no. 60473N," in Fourth International Conference on Photonics and Imaging in Biology and Medicine, Pts 1 and 2. vol. 6047, K. Xu, Q. Luo, D. Xing, A. V. Priezzhev, and V. V. Tuchin, Eds., ed, 2006, pp. N473-N473.

[31] R. Othonos and K. Kalli, Fiber Bragg Gratings: Fundamentals and applications in telecommunications and sensing. Norwood, MA: Artech House, 1999.

[32] J. W. Arkwright, N. G. Blenman, I. D. Underhill, S. A. Maunder, M. M. Szczesniak, P. G. Dinning, et al., "In-vivo demonstration of a high resolution optical fiber manometry catheter for diagnosis of gastrointestinal motility disorders," Opt. Exp., vol. 17, pp. 45004508,2009

[33] S. A. Wade, D. P. Attard, and P. R. Stoddart, "Analysis of transmission mode of a matched fiber Bragg grating interrogation scheme," Appl. Opt., vol. 49, pp. 4498-4505, 2010.

[34] T. G. Landry, A. K. Wise, J. B. Fallon, and R. K. Shepherd, "Spiral ganglion neuron survival and function in the deafened cochlea following chronic neurotrophic treatment," Hear. Res., vol. 282, pp. 303-313, 2011.

[35] R. K. Shepherd, A. Coco, S. B. Epp, and J. M. Crook, "Chronic depolarization enhances the trophic effects of brain-derived neurotrophic factor in rescuing auditory neurons following a sensorineural hearing loss," J. Comp. Neurol., vol. 486, pp. 145158,2005

[36] R. K. Shepherd and J. Xu, "A multichannel scala tympani electrode array incorporating a drug delivery system for chronic intracochlear infusion," Hear. Res., vol. 172, pp. 92-98, Oct 2002.

[37] R. T. Richardson and A. K. Wise, "Polypyrrole-coated electrodes for the delivery of charge and neurotrophins to cochlear neurons," Biomaterials, vol. 30, pp. 2614-2624, 2009. 
[38] R. Shepherd, K. Verhoeven, J. Xu, F. Risi, J. Fallon, and K. Wise, "An improved cochlear implant electrode array for use in experimental studies," Hear. Res., vol. 277, pp. 20-27, 2011.

[39] A. A. Eshraghi, N. W. Yang, and T. J. Balkany, "Comparative study of cochlear damage with three perimodiolar electrode designs," Laryngoscope, vol. 113, pp. 415-419, 2003.

[40] T. Ishii, M. Takayama, and Y. Takahashi, "Mechanical-properties of human round window, basilar and reissners membranes," Acta Oto-Laryngol., pp. 78-82, 1995.

[41] S. A. Wade, D. I. Forsyth, K. T. V. Grattan, and Q. Guofu, "Fiber optic sensor for dual measurement of temperature and strain using a combined fluorescence lifetime decay and fiber Bragg grating technique," Rev. Sci. Instrum., vol. 72, pp. 3186-3190, 2001.

[42] W. Jin, W. C. Michie, G. Thursby, M. Konstantaki, and B. Culshaw, "Simultaneous measurement of strain and temperature: error analysis," Opt. Eng., vol. 36, pp. 598-609, 1997.

[43] J. Zhang, S. Bhattacharyya, and N. Simaan, "Model and parameter identification of friction during robotic insertion of cochlearimplant electrode arrays," in IEEE International Conference on Robotics and Automation, Vols 1-7, 2009, pp. 3528-3533.

[44] O. Majdani, D. Schurzig, A. Hussong, T. Rau, J. Wittkopf, T. Lenarz, et al., "Force measurement of insertion of cochlear implant electrode arrays in vitro: comparison of surgeon to automated insertion tool," Acta Oto-Laryngol., vol. 130, pp. 31-36, Jan 2010.

[45] T. S. Rau, A. Hussong, M. Leinung, T. Lenarz, and O. Majdani, "Automated insertion of preformed cochlear implant electrodes: evaluation of curling behaviour and insertion forces on an artificial cochlear model," Int. J. CARS, vol. 5, pp. 173-181, Mar 2010.

[46] C. A. Todd, F. Naghdy, and M. J. Svehla, "Force application during cochlear implant insertion: An analysis for improvement of surgeon technique," IEEE Trans. Biomed. Eng., vol. 54, pp. 12471255, Jul 2007.

[47] G. Kontorinis, T. Stoeve, T. Lenarz, and G. Paasche, "Insertion speed of cochlear implant electrode arrays: Correlation with electrode type and surgeon's experience," in Proceedings of the Thirty-Fourth Annual Midwinter Resarch Meeting of the Association for Research in Otolaryngology, Baltimore, MD, 2011.

[48] H. N. Kha, B. K. Chen, G. M. Clark, and R. Jones, "Stiffness properties for Nucleus standard straight and contour electrode arrays," Med. Eng. Phys., vol. 26, pp. 677-685, Oct 2004.

[49] J. Patrick and J. McFarlane, "Characterization of mechanical properties of single electrodes and multielectrodes," Ann. Otol. Rhinol. Laryngol., vol. 96, pp. 46-48, 1987.

[50] N. Mohammad, W. Szyszkowski, W. J. Zhang, E. I. Haddad, J. Zou, W. Jamroz, et al., "Analysis and development of a tunable fiber Bragg grating filter based on axial tension/compression," $J$. Lightwave Technol., vol. 22, pp. 2001-2013, Aug 2004.

[51] H. Kha and B. Chen, "Finite element analysis of damage by cochlear implant electrode array's proximal section to the basilar membrane," Otol. Neurotol., vol. 33, pp. 1176-1180, 2012.

[52] B. K. Chen, G. M. Clark, and R. Jones, "Evaluation of trajectories and contact pressures for the straight nucleus cochlear implant electrode array - a two-dimensional application of finite element analysis," Med. Eng. Phys., vol. 25, pp. 141-147, Mar 2003.

[53] S. J. Rebscher, M. Heilmann, W. Bruszewski, N. H. Talbot, R. L. Snyder, and M. M. Merzenich, "Strategies to improve electrode positioning and safety in cochlear implants," IEEE Trans. Biomed. Eng., vol. 46, pp. 340-352, Mar 1999. 


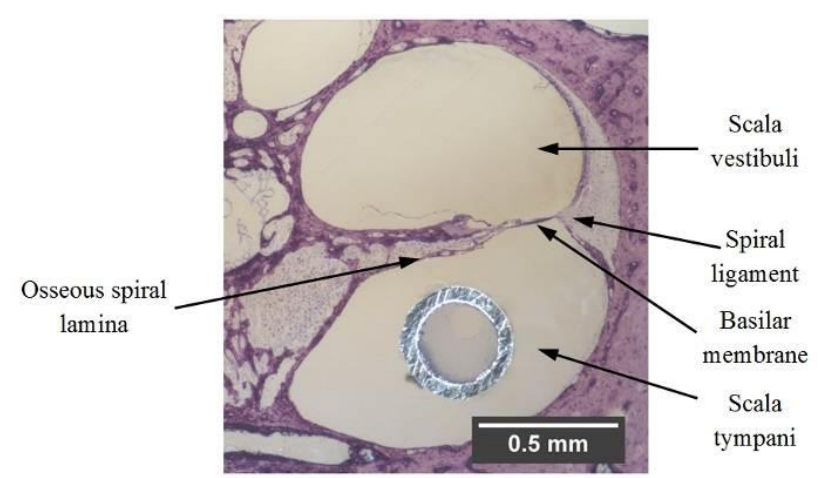

Fig. 1. Histological cross-section of a guinea pig cochlear showing some of the major anatomical features. Note: the circular ring in the scala tympani region is a platinum electrode which was cross-sectioned during the sample preparation.

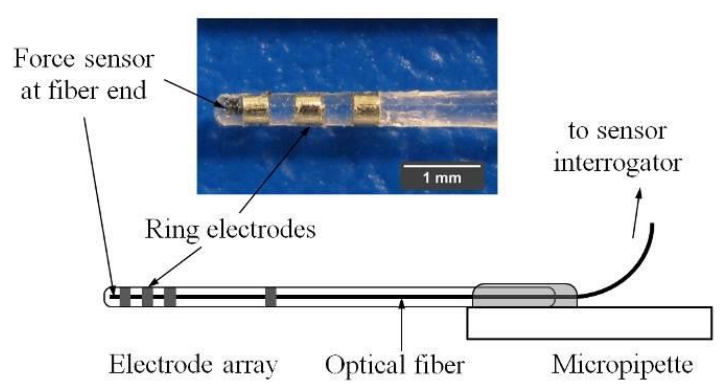

Fig. 2. Diagram illustrating the electrode assembly containing the optical fiber force sensor, attached to a micropipette for use in an insertion trial.

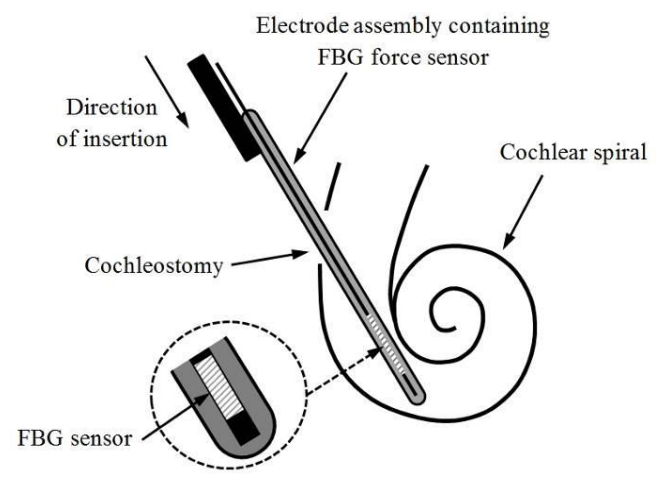

Fig. 3. Diagram illustrating details of the insertion of the electrode array and the location of the optical fiber force sensor at the tip of the array (Note: not to scale).
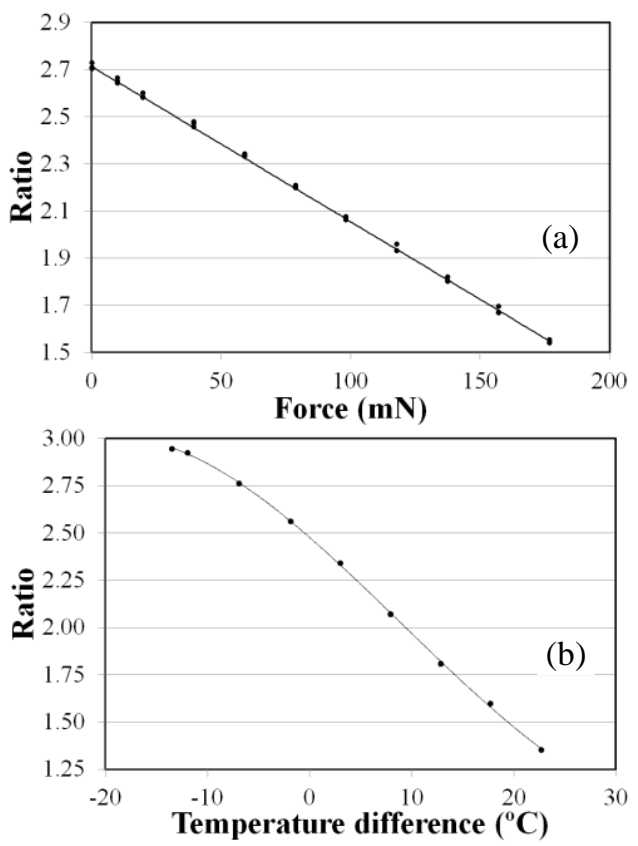

Fig. 4. Examples of force (a) and temperature (b) calibration data for the optical fiber force sensor. The solid line in the force calibration data is a linear fit, while a $3^{\text {rd }}$ order polynomial has been fitted to the temperature calibration data to help show the trend. 


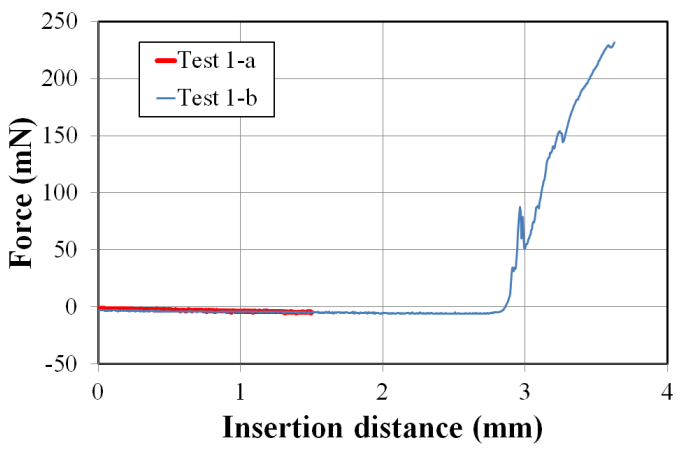

Fig. 5. Forces measured during an atraumatic (1-a, insertion stopped at $+1.5 \mathrm{~mm})$ and a traumatic (1-b, insertion stopped at $+3.63 \mathrm{~mm}$ ) insertion test.

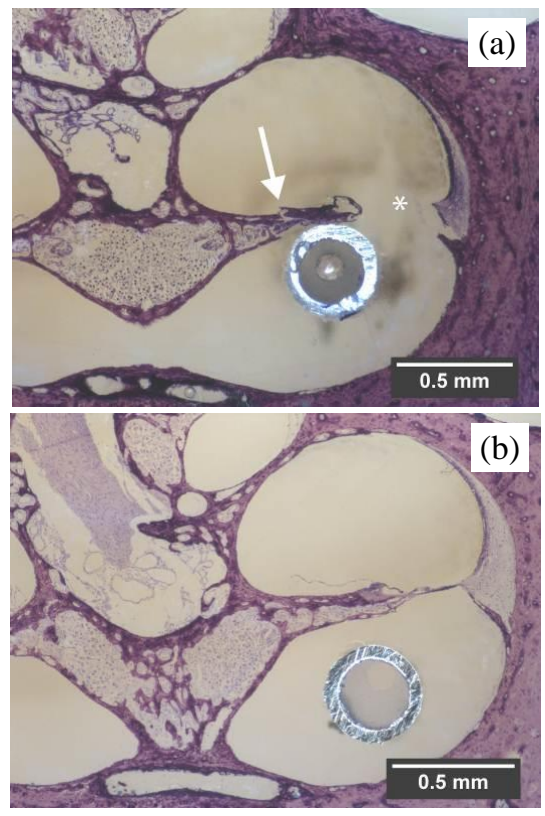

Fig. 6. Examples of histological images of (a) traumatic and (b) atraumatic insertions, from tests 1-b and 1-c respectively. The location of the electrode in each of the images is clearly identified by the circular platinum rings which have been cross-sectioned during the sample preparation. Areas of trauma are highlighted by the arrow and star in (a).

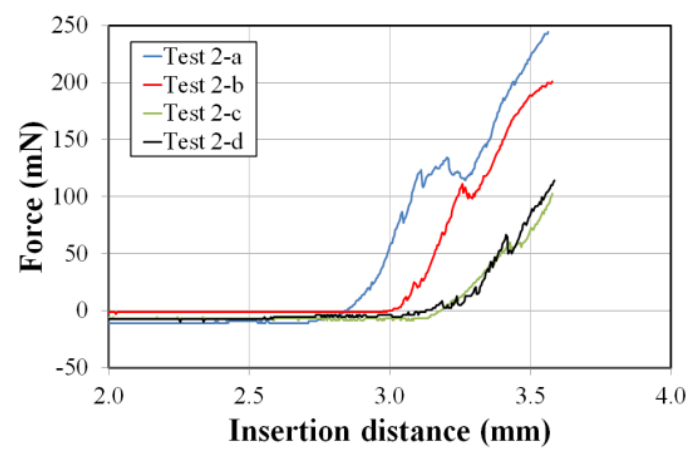

Fig. 7. Forces measured during insertion experiment \#2, where the insertions were stopped at $\sim+3.5 \mathrm{~mm}$.

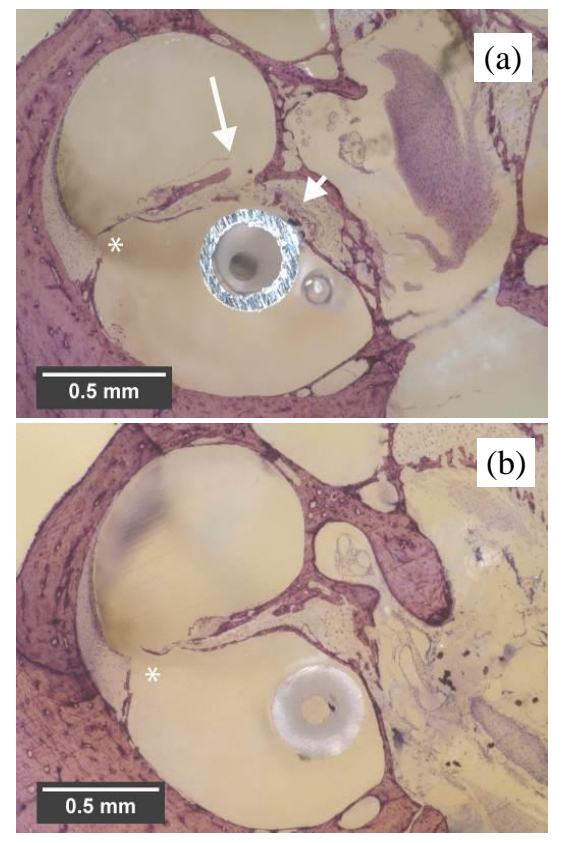

Fig. 8. Examples of histological images of insertions performed in experiment \#2 with maximum measured forces of (a) $244 \mathrm{mN}$ and (b) $102 \mathrm{mN}$. Again the electrode array positions can be clearly seen. Areas of trauma are highlighted by the arrows and stars. 


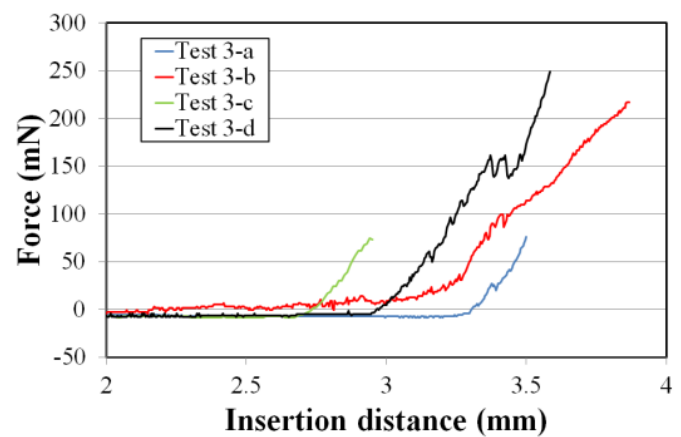

Fig. 9. Forces measured during insertion experiment \#3, in which insertions were stopped at either $\sim 75 \mathrm{mN}$ or $\sim 225 \mathrm{mN}$.

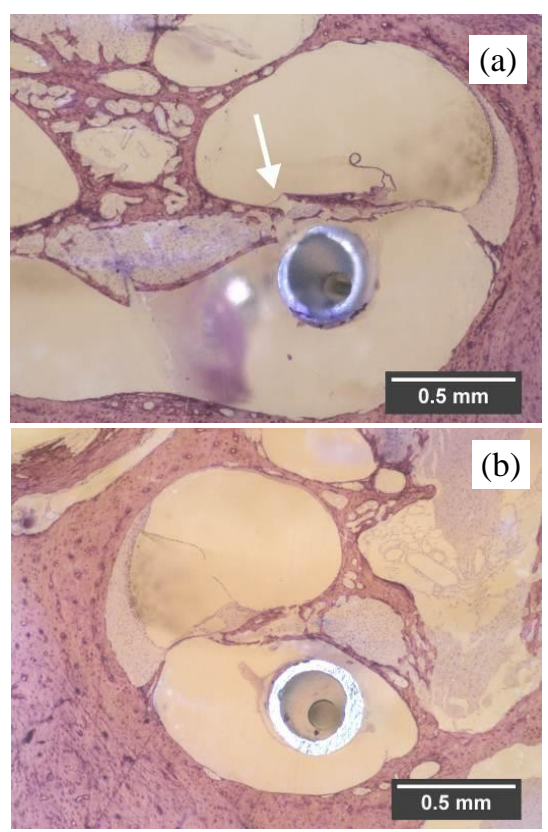

Fig. 10. Examples of histological images of insertions performed in experiment \#3 with maximum measured forces of (a) $217 \mathrm{mN}$ and (b) $75 \mathrm{mN}$. Again the electrode array positions can be clearly seen. An area of trauma is highlighted by the arrow in (a).

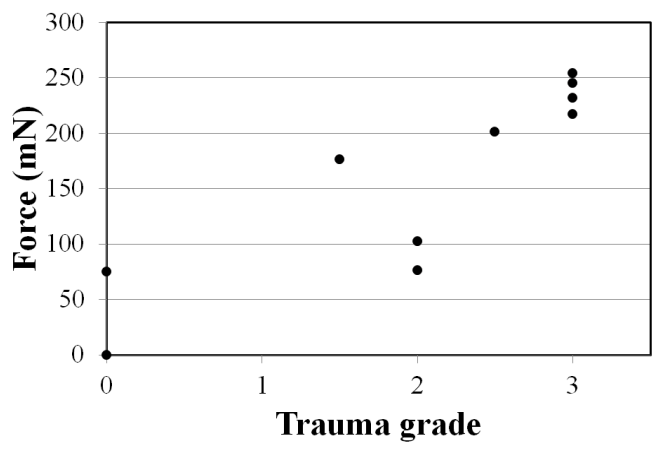

Fig. 11. Trauma gradings for each of the insertion tests, where histological examination was possible, and the

TABLE I

FORCE MEASUREMENTS AND INSERTION DAMAGE

\begin{tabular}{ccc}
\hline \hline Insertion Test & Maximum Force $(\mathrm{mN})$ & Trauma Grade \\
\hline $1-\mathrm{a}$ & 0 & N/A* \\
$1-\mathrm{b}$ & 232 & 3 \\
$1-\mathrm{c}$ & 0 & 0 \\
$1-\mathrm{d}$ & 176 & $1-2$ \\
\hline $2-\mathrm{a}$ & 245 & 3 \\
$2-\mathrm{b}$ & 201 & $2-3$ \\
$2-\mathrm{c}$ & 102 & 2 \\
$2-\mathrm{d}$ & 114 & N/A* \\
\hline $3-\mathrm{a}$ & 76 & 2 \\
$3-\mathrm{b}$ & 217 & 3 \\
$3-\mathrm{c}$ & 75 & 0 \\
$3-\mathrm{d}$ & 254 & 3 \\
\hline \hline
\end{tabular}

N/A* - issues with the histology of the samples from these insertions prevented estimations of insertion damage. 


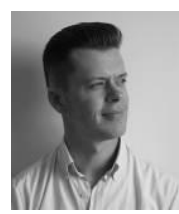

Scott A. Wade graduated from Victoria University of Technology (Melbourne, Australia) with a B.Sc. (Hons.) in applied physics in 1994, and was subsequently awarded a Ph.D. in 2001 for research on optical fiber-based temperature sensors.

$\mathrm{He}$ is currently a Senior Lecturer in Biomedical Engineering in the Faculty of Engineering and Industrial Sciences at Swinburne University of Technology (Melbourne, Australia). He has experience in the research and development of various types of optical fiber sensors for a wide range of applications. His current research interests include biomedical applications of optical fibers for light delivery and sensing, the development of optical fiber sensors, corrosion sensing and microbiologically influenced corrosion.

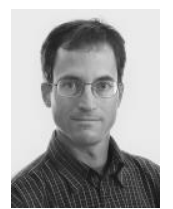

Paul R. Stoddart graduated with a B.Sc. degree (Hons) in physics and a Ph.D. degree in laser spectroscopy from the University of the Witwatersrand, South Africa. After working on industry-focused surface science and microanalysis problems in a national lab for three years, he joined Swinburne University of Technology, Melbourne, Australia, in 2001. He is currently an Associate Professor in Biomedical Engineering at Swinburne. His current interests include applied optics and biophotonics, with projects in the areas of fiber optic sensors, Raman spectroscopy and laser nerve stimulation.

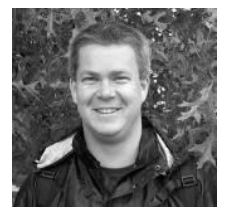

James B. Fallon (M'04) received the B.Sc. (1998), B.E.(Hons) (1999) and $\mathrm{PhD}$ (2002) from Monash University, Melbourne, Australia. He is a senior research fellow at The Bionic Ear Institute, and his current research focus is on deafness and cochlear implant induced changes in the central auditory pathway.

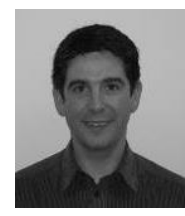

Andrew D. Wise graduated from Monash University (PhD) in 2001. He was a post-doctoral research Fellow at the Bionics Institute (2001-2003). He moved to Bristol UK where he took up a post-doctoral position (2003-2006) before returning to Melbourne in 2006 to continue research at the Bionics Institute. His research interests are focused on the use of drug delivery to prevent degeneration of cochlear cells following deafness, with or without cochlear implantation, and examining the effects of this treatment on auditory function.

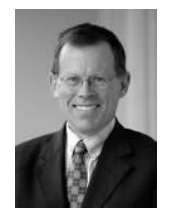

Robert K. Shepherd (M'09) was born in Portland, Victoria, Australia in 1953. He graduated with a BSc degree in physics from Deakin University in 1976, a Grad Dip Education from Hawthorn State College in 1977 and a PhD in otolaryngology from the University of Melbourne in 1987 examining the safety and efficacy of cochlear implants.

$\mathrm{He}$ is currently Director of the Bionics Institute, Melbourne Australia and Professor of Medical Bionics, The University of Melbourne. His current research interests include the development of retinal prostheses and neural prostheses for neurological disorders, as well as contributing to improvements in cochlear implant design.

Dr Shepherd is a member of the Association for Research in Otolaryngology, Association for Research in Vision and Ophthalmology, the Australian Neuroscience Society and is a life member of the International Functional Electrical Stimulation Society.

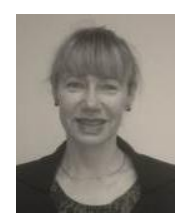

Natalie L. James graduated from the University of Sydney (Australia) with a B.Sc (Hons) degree in biochemistry and mathematics. She has M. Biomed. E. and Ph.D. degrees from the University of New South Wales (Sydney, Australia).

She was awarded a National Heart Foundation of Australia Fellowship to investigate endothelial cell recognition of blood flow at Georgia Institute of Technology and Emory University in Atlanta. She has developed materials and assessed the biocompatibility of cardiovascular and hearing devices. At Cochlear Limited she has worked on the biological interface with the implant, biofilm and antimicrobial infection. She has research interests in polymeric encapsulation of active implantable devices, applications of lasers, optical fibers and thin film coating technologies. 